\title{
Higher Heart-type Fatty Acid Binding Protein Levels are Related to More Severe and Extensive Coronary Atherosclerosis in Patients with Acute Myocardial Infarction
}

\author{
(1) Togay Evrin¹, (1) Aycan Fahri Erkan², (1) Berkay Ekici², (1) Selda Demirtaș3 ${ }^{3}$, (1) Șule Korkmaz ${ }^{4}$, (1) Atilla Korkmaz ${ }^{5}$, \\ (D) Eylem Kuday Kaykısız' ${ }^{6}$, (1) Burak Katipoğlu1
}

1Department of Emergency Medicine, Ufuk University Faculty of Medicine, Ankara, Turkey

2Department of Cardiology, Ufuk University Faculty of Medicine, Ankara, Turkey

${ }^{3}$ Department of Medical Biochemistry, Ufuk University Faculty of Medicine, Ankara, Turkey

${ }^{4}$ Clinic of Cardiology, VKV American Hospital, i̇stanbul, Turkey

${ }^{5}$ Department of General Surgery, Ufuk University Faculty of Medicine, Ankara, Turkey

${ }^{6}$ Clinic of Emergency Medicine, Bitlis State Hospital, Bitlis, Turkey

\begin{abstract}
Aim: Heart-type fatty acid binding protein (H-FABP), an early marker of cardiac necrosis, is released rapidly from myocardium as a consequence of ischemic injury. We hypothesized that more severe and extensive coronary atherosclerosis would result in more pronounced myocardial injury and necrosis in patients with acute myocardial infarction (AMI). Therefore, we sought the relationship between serum $\mathrm{H}$-FABP levels and the severity and extent of coronary artery disease (CAD) assessed using the Gensini score.

Materials and Methods: Fifty patients with AMI who underwent invasive coronary angiography were divided into 2 groups according to the angiographic Gensini score, namely the moderate to severe CAD group (group 1) and the mild CAD group (group 2). A point of care test and Cardiodetect Quant device were used to detect whether H-FABP was positive and the quantitative measurements. The data obtained from this study were evaluated using the PASW statistic programme.

Results: Mean serum H-FABP concentration was significantly higher in group 1 when compared to that in group 2 . Furthermore, a strongly positive correlation was found between the Gensini score and serum H-FABP levels.

Conclusion: The findings of our study suggest that the quantity of myocardial necrosis demonstrated by serum H-FABP levels is higher in patients with AMI who have more severe and extensive CAD. H-FABP levels are also positively correlated to the Gensini score. We propose that H-FABP, an early marker of myocardial necrosis, may also provide a clue about the severity and extent of CAD, especially in the setting of AMI.
\end{abstract}

Keywords: Myocardial infarction, coronary artery disease, Gensini score, Heart-type fatty acid binding protein

\section{Introduction}

Cardiovascular diseases are increasingly becoming a major cause of mortality and morbidity worldwide (1). Acute coronary syndrome (ACS), usually formed by the rupture of atherosclerotic plaque in the coronary arteries or total occlusion of the coronary artery Subtotal thrombus clinical emergency that occur as a result of table (2-4). Early diagnosis of axillary patients is very important in terms of directing their treatment. Mortality and morbidity will be positively affected by early diagnosis and timely treatment of thrombolytic therapy, and complications that may occur during the follow-up of the disease will be easier to control $(5,6)$. Evidence has been gathered that inflammation plays an important role in the onset and progression of atherosclerosis, and all of these 
suggest that inflammation markers may help predict individual risk for cardiovascular disease (7). The present risk factors may explain only half of all cases of coronary artery disease (CAD) and long-term cardiac death and complications. This inadequacy has sparked an intense interest in the identification of new biomarkers that could contribute to the prediction power of classical risk factors (8). A marker of acute myocardial infarction, acute myocardial infarction (AMI)-induced sarcoidemal injury, cardiac fatty acid binding protein (H-FABP), begin to increase within 1-3. hours, reaching the highest level within 6-8 hours and within 24-36 hours return to normal levels. These plasma kinetics and oscillation properties and various clinical studies have shown H-FABP performance superior to myoglobin, Creatine kinase (CK), creatine kinase heart type (CK-MB) and troponin in early AMI diagnosis (9-11). A number of scoring systems based on coronary angiography images have been developed to determine the severity of CAD. Gensini score is a scoring system that have been developed for this purpose and used to evaluate the severity of coronary artery disease. There are 2 coronary angiography scores: vascular scoring and stenosis scoring. These techniques have been described by Gensini. According to the angiographic degree of stenosis, narrowing between $0-25 \%, 25-50 \%, 50-75 \%$, 75-90\%, 90-99\%, and 100\%, stenosis is scored as, 1, 2, 4, 8, 16, and 32 points, respectively (12). CAD is the most common cause of death in the United States and the most common cause of death in the United States. We considered that H-FABP, an earlystage cardiac necrosis marker, may be informative in terms of severity and prevalence by reviewing the literature .In this study, we aimed to investigate the relationship between $\mathrm{H}$-FABP and Gensini scores in patients with high cardiac troponin, CK and CK$\mathrm{MB}$ with cardiac necrosis markers.

\section{Materials and Methods}

This study was initiated after obtaining the necessary permission from the local Ethics Committee of Ufuk University Faculty of Medicine. A retrospective study of 735 patients who underwent coronary artery bypass grafting for treatment of coronary artery bypass grafting according to the guidelines of American Society of Cardiology (ACC/AHA) from the Emergency Department of Ufuk University between 05.12.2008 and 24.02.2011 was performed on 735 patients. The patients who had previous cardiopulmonary resuscitation and fibrinolytic treatment, and percutaneous coronary intervention, patients with coronary artery intensive care, those with trauma within 3 days before admission, patients with unstable angina pectoris according to ACC/(USAP), the patients with chronic renal failure and serum creatinine level of $1.5 \mathrm{mg} / \mathrm{dL}$ the patients who underwent coronary intensive care unit through PTCA or CABG within 1 month, the patients who had STEMI or NSTEMI within 1 month before admission, the patients with pulmonary thromboembolism, patients with AMI, and patients younger than 18 years were not included in this study $(13,14)$.

According to guidelines of ACC/AHA , the patients with acute coronary syndromes with ST segment elevation and non-ST segment elevation without ST segment elevation were included in the study. All of the patients biochemical parameters, ECG information, the admission of patients until the day they are discharged from the track at highest cardiac necrosis markers (CK-MB, Troponin-T, H-FABP) levels and coronary artery disease with coronary angiography Gensini scores by examining the scores of the seriousness of the form of work have been recorded $(13,14)$. A test based on the rapid chromatographic immunoassay method called CardioDetect Med was used by the manufacturer (Rennesens GmbH Berlin, Germany) for the measurement of $\mathrm{H}$-FABP values. Coronary angiographies were interpreted with the Gensini score assessing CAD severity (15). Patients are considered to have mild coronary atherosclerosis with a score of 1-20, and severe coronary atherosclerosis with a score of 20 according to Gensini scores (16). Patients included in the study were divided into two groups: those with Gensini scores below 20 (group 1), those with 20 and above (group 2).

\section{Statistical Analysis}

In comparison; the Kolmogorov-Smirnov test, which is a normal distribution test, was performed, and the normal distribution variables were evaluated by independent sample T test. MannWhitney $U$ test was used for the variables without normal distribution, and chi-square test was used for categorical variables. Relationships between Gensini scores of H-FABP, CK-MB and troponin- $T$ values were investigated by Spearman correlation analysis. The data obtained in this study were evaluated with the help of PASW statistical package program. A p value of less than 0.05 was considered statistically significant.

\section{Results}

All of the patients, 50 patients, were included diagnosed as AMI according to the AHA $(13,14)$. In our study, the patients the Gensini scores of whom were lower than 20 (group 1), 16 (32\%), and the Gensini scoress were higher than 20 (group 2) and 34 (68\%) were ill. Of the 50 patients included in the study, $38(76 \%)$ were male and $12(24 \%)$ were female. The average age of the 50 patients included in the study was $64.2 \pm 11.498$ (minimum: 40-maximum: 87) years. There was no significant difference in the distribution of age and sex between the groups in our study $(p>0.05)$. Of the 50 patients included in the study, 36 (72\%) were diagnosed as NSTEMI and 14 (28\%) were diagnosed as STEMI. Plasma levels of FABP, CK-MB, and Troponin-T for the groups are shown in Table 1. 
Table 1. Plasma levels of fatty acid binding protein, CK-MB, Troponin-T according to groups

\begin{tabular}{|c|c|c|c|c|c|c|}
\hline & & & & & & Independent samples T test \\
\hline & Severity of KAH & $\mathrm{N}$ & Mean & Minimum & Maximum & Sig. 2 tailed \\
\hline \multirow{2}{*}{ FABP } & Under 20 & 16 & 13.0313 & 7 & 23 & 0.000 \\
\hline & 20 and over 20 & 34 & 55.1471 & 13 & 100 & \\
\hline CK-MB & Under 20 & 16 & 66.4238 & 5.21 & 332.6 & 0.277 \\
\hline \multirow{2}{*}{ Troponin T } & Under 20 & 16 & 2.0112 & 0.04 & 12.61 & 0.172 \\
\hline & 20 and over 20 & 34 & 3.8253 & 0.11 & 20.81 & \\
\hline
\end{tabular}

FABP: Fatty acid binding protein

Table 2. The correlation of plasma fatty acid binding protein levels and Gensini scores

\begin{tabular}{l|lll}
\hline \multirow{3}{*}{ Spearman's rho } & Correlation & & Gensini \\
\cline { 2 - 4 } & & $\mathrm{r}$ & 0.926 \\
\cline { 3 - 4 } & FABP & $\mathrm{p}$ & 0.000 \\
\cline { 3 - 4 } & & $\mathrm{N}$ & 50 \\
\hline
\end{tabular}

Table 3. The correlation of plasma CK-MB levels and Gensini scores

\begin{tabular}{l|lll}
\hline \multirow{2}{*}{ Spearman's rho } & Correlation & \multicolumn{1}{c}{ Gensini } \\
\cline { 2 - 4 } & \multirow{2}{*}{ CK-MB } & $r$ & 0.195 \\
\cline { 3 - 4 } & & $\mathrm{p}$ & 0.174 \\
\cline { 3 - 4 } & & $\mathrm{N}$ & 50 \\
\hline
\end{tabular}

Table 4. The correlation of plasma Troponin-T levels and Gensini scores

\begin{tabular}{l|lll}
\hline \multirow{3}{*}{ Spearman's rho } & Correlation & \multicolumn{1}{c}{ Gensini } \\
\cline { 2 - 4 } & \multirow{2}{*}{ Troponin-T } & $\mathrm{r}$ & 0.142 \\
\cline { 3 - 4 } & & $\mathrm{p}$ & 0.325 \\
\cline { 3 - 4 } & & $\mathrm{N}$ & 50 \\
\hline
\end{tabular}

According to the severity of CAD, there was a statistically significant difference between the FABP levels of the patients in group 2 and the FABP levels of the patients in group $1(p<0.05)$. As to the Troponin-T values of the patients in group 2 according to the severity of CAD, There was no statistically significant correlation between the levels of Troponin-T of the patients $(p>0.05)$. There was no statistically significant correlation between the CK-MB levels of the patients in group 2 and the CK-MB levels of the patients in group 1 ( $p>0.05)$. Correlation of plasma FABP, CK-MB, Troponin-T levels and Gensini scores of patients is shown in Tables 2, 3, 4. There was a statistically significant positive correlation between the FABP levels of all patients with ACS who were included in the study and the Gensini scores calculated after coronary

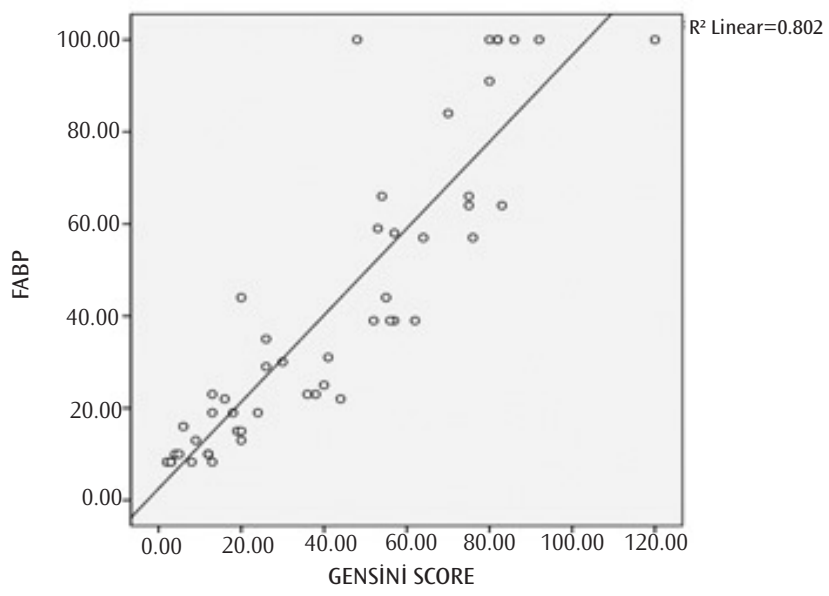

Figure 1. Correlation graph of plasma fatty acid binding protein levels and Gensini scores of patients

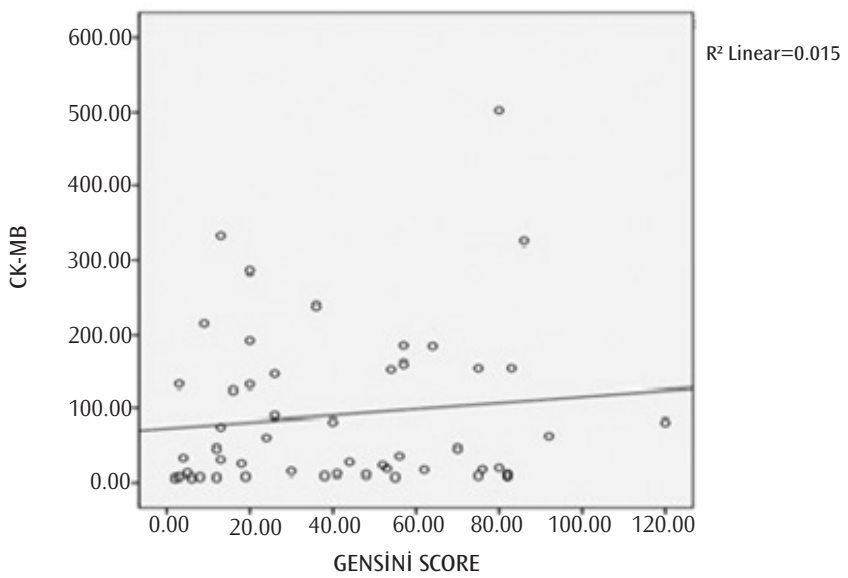

Figure 2. Correlation graph of plasma CK-MB levels and Gensini scores of patients

angiography $(p<0.005)$. Correlation Graph of plasma FABP levels and Gensini scores of patients is shown in Figure 1. Correlation Graph of plasma CK-MB levels and Gensini scores of patients is shown in Figure 2. Correlation Graph of plasma Troponin-T levels and Gensini scores of patients is shown in Figure 3. 


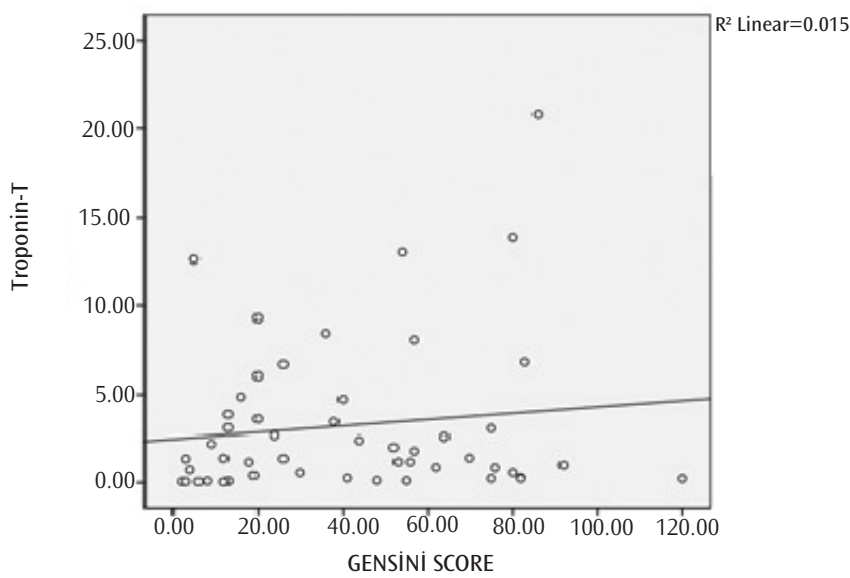

Figure 3. Correlation graph of plasma Troponin-T levels and Gensini scores of patients

\section{Discussion}

$\mathrm{H}$-FABP, an indicator of AMI-associated sarcolemmal injury, increases in $1-3^{\text {rd }}$. hours of $A M I$, reaches the highest level within 6-8 hours, and returns to normal levels within 24-36 hours (9).

The relationship between plasma $\mathrm{H}$-FABP and the severity of coronary artery disease is not fully known, though it is known that H-FABP is an important cardiac marker for ACS with plasma kinetics and with its release characteristics. Up to date, no study in the literature has shown a relationship between the severity of CAD and the serum levels of H-FABP, CK-MB, and Troponin-T in patients with ACS, whose cardiac necrosis markers are positive. However, there are studies investigating the relationship between serum H-FABP values and prognosis of patients with ACS (17-21). In addition, there was no study in the literature regarding the correlation between serum H-FABP, CK-MB, Troponin-T levels and Gensini scores in patients with ACS in which cardiac necrosis markers were positive.

In a study conducted by O'Donoghue et al. (18) the patients diagnosed with ACS were those with a serum H-FABP value of 8 $n g / d L$ and above $(n=332)$ and those with serum $\mathrm{H}-\mathrm{FABP}<8 \mathrm{ng} / \mathrm{dL}$ $(n=1955)$ were separated into two groups and monitored for 10 months. There was a statistically significant difference between these two groups in terms of developing cardiac death, $\mathrm{MI}$, and CHF development within 10 months $(p<0.001)(18)$.

In the same study conducted by O'Donoghue et al. (18) the patients diagnosed with ACS were those with serum H-FABP $>16$ $n g / d L(n=166)$, those with serum H-FABP values between 8 and $16(n=166)<8 \mathrm{ng} / \mathrm{dL}(\mathrm{n}=1955)$ were separated and monitored for 10 months. There was a statistically significant difference $(p<0.001)$ in the group with serum H-FABP $>16 \mathrm{ng} / \mathrm{dL}$ in terms of developing cardiac-induced death, subsequent MI transmission and CHF development within 10 months compared to the other groups (18).

According to the retrospective study performed by Suzuki et al. (19) 90 patients with ACS were qualitatively divided into two groups as H-FABP positive $(n=56)$ and negative $(n=34)$ and a statistically significant difference was found by the Cox Proportional Risk Method for developing MI and decompensated CHF and subsequent MI $(p<0.005)$.

According to the prospective study performed by McCann et al., (20) the patients admitted to the coronary intensive care unit with ischemic chest pain $(n=664)$ were followed up for 1 year. They were into separated 2 groups, as experienced 1 cardiac event and as 1 experienced no cardiac event. There was a statistically significant difference between H-FABP and Troponin-T values $(p<0.001, p<0.001)$.

According to a prospective study conducted by Viswanathan et al. (21), 955 patients with suspected ACS, who were referred to emergency services, were followed for 48 weeks. Patients were divided into 4 groups according to serum H-FABP values. Serum $\mathrm{H}-\mathrm{FABP}<3.27 \mathrm{ng} / \mathrm{dL}$ group-1, H-FABP 3.27-6.48 ng/dL group 2, H-FABP 6.49-12.77 ng/-FABP\&gt; 12.77 group 4. There was a statistically significant difference between group 3 and group 4 and group 1 according to the number of patients who had cardiac death and MI after 48 weeks $(p<0.0001, p<0.0001)$. There was also a statistically significant difference between group 4 and the other groups according to whether patients had a history of CAD $(p<0.001)(21)$. This shows us that the CAD story of the patients is a significant risk factor for evaluating the prognosis of the CAD as it is in our study.

In the same study of Viswanathan et al. (21) patients with positive troponin- $T$ values were excluded and the remaining 756 patients were re-divided into 4 groups according to serum H-FABP values. There was a statistically significant difference between group-3 and group- 4 and group- 1 according to the number of patients who had cardiac death and MI at 48 weeks $(p<0.0001, p<0.0001)$ (21). In the same study of Viswanathan et al. (21) 955 patients with suspected ACS, who were admitted to emergency services, were followed up for 48 weeks. Patients were divided into 4 groups according to serum troponin-T values. Serum troponin-T group-1 between 0.00-0.02 $\mu \mathrm{g} / \mathrm{L}$, group-2 between troponin- $\mathrm{T}$ 0.03-0.08 ug/L, group-2 between troponin-T 0.09-3.04 group-3 and troponin- $\mathrm{T}>3.04 \mu \mathrm{g} / \mathrm{L}$ group- 4 . There was a statistically significant difference between group-3 and group-4 and group-1 according to the number of patients who had cardiac death and $\mathrm{Ml}$ at 48 weeks $(p<0.0001, p<0.0001)(21)$. These studies carried out by Viswanathan et al. (21) indicate that H-FABP is a prognostic serum biomarker, independent of troponin-T, while emphasizing 
that serum $\mathrm{H}$-FABP values and troponin-T values are important for assessing long-term mortality in CAD.

Ishii et al. (22) reported that patients who were admitted to the hospital with ACS diagnosis $(n=328)$ were divided into two groups: group-1 at $9.8 \mathrm{ug} / \mathrm{L}$ and group-2 at $9.8 \mathrm{ug} / \mathrm{L}$. They separated the group and followed the patients for 6 months. At 6 months, there was a statistically significant difference between the group with cardiac mortality H-FABP value above $9.8 \mu \mathrm{g} / \mathrm{L}$ and the other group $(p<0.0001)(22)$. In the same study conducted by Ishii et al., (22) it was determined that there was a statistically significant difference in cardiac-induced mortality after 6 months $(p<0.0001)$ between patients with elevated troponin- $T$ and H-FABP and patients with elevated troponin-T alone) (22).

In our study, there was a numerical difference between the mean troponin-T values of the patient group with a high CAD severity (group 2) and the mean troponin-T values of the patient group with low CAD severity (group 1) (group $1=2.011 \mathrm{ng} / \mathrm{mL}$ group $2=3.825 \mathrm{ng} / \mathrm{mL}$ ). However, there was no statistically significant difference between the two groups in our study $(p>0.005)$. The knowledge in the literature that the troponin- $T$ can assess longterm mortality in CAD suggests that this study is related to the low number of patients in group 1 in our study, although it is statistically incompatible with our study in terms of statistically.

$\mathrm{H}$-FABP, a marker of AMI-associated sarcolemmal injury, increases in 1-3 $3^{\text {rd }}$. hours of AMI, reaches the highest level within 6-8 hours, and returns to normal levels within 24-36 hours (9). Although $\mathrm{H}-\mathrm{FABP}$ is an important cardiac marker for ACS with this plasma kinetics and oscillation feature, it is also a new cardiac marker in evaluating the long-term mortality and prognosis of patients with ACS in light of our study and these studies in the literature. There is a relationship between H-FABP and CAD severity in the data obtained from our studies and from literature studies.

\section{Study Limitations}

The most significant limitation of our study is our small sample size. It is necessary to support our findings with larger scale, prospective and multicenter studies.

\section{Conclusion}

There is a need for a non-invasive, easily reproducible technique that meets the clinical need to determine the severity of CAD and its prognosis early. In our study, serum H-FABP levels were significantly higher in patients with high CAD severity (group 2) and patients with low CAD severity (group 1). It was also determined that there was a significant positive correlation between Gensini score and serum H-FABP values in all patients with positive cardiac necrosis markers. There is a relationship between $\mathrm{H}-\mathrm{FABP}$ and $\mathrm{CAD}$ severity, suggesting that H-FABP can provide information about the prevalence and severity of $C A D$ even in the early period of cardiac damage. In conclusion, the relevant studies that use serum $\mathrm{H}$-FABP levels as a predictor of cardiovascular risk require more extensive, prospective studies to diagnose ACS patients, to determine the need for intervention, and to predict the severity of coronary artery disease.

\section{Ethics}

Ethics Committee Approval: Ufuk University, Faculty of Medicine, approval number: 15062012-5.

Informed Consent: Informed consent did not obtained from the participants due to retrospective nature of the study.

Peer-review: Externally peer-reviewed.

\section{Authorship Contributions}

Surgical and Medical Practices: T.E., A.F.E., Concept: B.E., S.D., Design: S.K., A.K., Data Collection or Processing: E.K.K., Analysis or Interpretation: B.K., Literature Search: B.E., A.K., Writing: T.E., E.K.K.

Conflict of Interest: No conflict of interest was declared by the authors.

Financial Disclosure: The authors declared that this study received no financial support.

\section{References}

1. Hennekens $\mathrm{CH}$. Increasing burden of cardiovascular disease. Current knowledge and future firections for research on risc factors. Circulation. 1998;97:1095-102.

2. Falk E, Shah PK, Fuster V. Coronary plaque distruption. Circulation. 1995:92:657-71.

3. Mann JM, Davies MJ. Vulnerable plaque. Relation of characteristics to degree of stenosis in human coronary arteries. Circulation. 1996;94:928-31.

4. Fuster V, Badimon L, Badimon JJ, Chesebro JH. The pathogenesis of coronary artery disease and acute coronary syndromes. N Engl ] Med. 1992;326:24250

5. Solinas L, Raucci R, Terrazzino S, Moscariello F, Pertoldi F, Vajto S, et al. Prevalence, clinical characteristics, resource utilization and outcome of patients with acute chest pain in theemergency department. A multicenter, prospective, observational study innorth-eastern Italy. Ital Heart J. 2003;4:318-24

6. Karlson BW, Wiklund I, Bengston A, Herlitz J. Prognosis and symptoms one year after discharge from the emergency department in patients with acute chestpain. Chest. 1994;105:1442-7.

7. Kelly AE, Gensini GG. Coronary arteriography and left-heart studies. Heart Lung. 1975;4:85-98.

8. de Korte CL, van der Steen AF, Cepedes El, Pasterkamp G, Carlier SG, Mastik $F$, et al. Characterization of plaque components and vulnerability with intravascular ultrasound elastography. Phys Med Biol. 2000;45:1465-75.

9. Early Diagnostic Performance of Heart-Type Fatty Acid Binding Protein in Suspected AcuteMyocardial Infarction: Evidence From a Meta-Analysis of Contemporary Studies. Xu LQ, Yang YM, Tong H, Xu CF. Heart Lung Circ. 2018;27:503-12. 
10. Mad P, Domanovits H, Fazelnia C, Stiassny K. Human heart-type fattyacid-binding protein as a point-of-care test in the early diagnosis of acute myocardial infarction. QJM. 2007;100:203-10.

11. Bruins Slot MH, van der Heijden GJ, Rutten FH. Heart-type Fatty acid-binding protein in Acute Myocardial infarction Evaluation (FAME): background and design of a diagnostic study in primary care. BMC Cardiovasc Disord. 2008;8:8.

12. Içli A, Gök H, Altunkeser BB, Ozdemir K, Gürbilek M, Gederet YT, Sökmen G. Evaluation of "admission index of insulin resistance (AIRI)" as an early stage risk predictor in nondiabetic acute coronary syndromes. Anadolu Kardiyol Derg. 2002;2:194-201.

13. Levine GN, Bates ER, Blankenship JC, Bailey SR, Bittl JA, Cercek B, et al. 2015 ACC/AHA/SCAI Focused Update on Primary Percutaneous Coronary Intervention for Patients With ST-Elevation Myocardial Infarction: An Update of the 2011 ACCF/AHA/SCAI Guideline for Percutaneous Coronary Intervention and the 2013 ACCF/AHA Guideline for the Management of ST-Elevation Myocardial Infarction: A Report of the American College of Cardiology/ American Heart Association Task Force on Clinical Practice Guidelines and the Society for Cardiovascular Angiography and Interventions. Circulation. 2016;133:1135-47

14. Ibanez B, James S, Agewall S, Antunes MJ, Bucciarelli-Ducci C, Bueno H,et al. 2017 ESC Guidelines for the Management of Acute Myocardial Infarction in Patients Presenting With ST-Segment Elevation: The Task Force for the Management of Acute Myocardial Infarction in Patients Presenting With STSegment Elevation of the European Society of Cardiology (ESC). Eur Heart J. 2018;39:119-77.

15. Gensini GG. A more meaningful scoring system for determining the severity of coronary heart disease. Am J Cardiol. 1983:51:606.
16. Amsterdam EA, Wenger NK, Brindis RG, Casey DE Jr, Ganiats TG, Holmes DR Jr, et al. 2014 AHA/ACC Guideline for the Management of Patients with NonST-Elevation Acute Coronary Syndromes: a report of the American College of Cardiology/American Heart Association Task Force on Practice Guidelines. J Am Coll Cardiol. 2014;64:e139-e228.

17. Oishi Y, Wakatsuki T, Nishikado A, Oki T, Ito S. Circulating adhesion molecules and severity of coronary atherosclerosis. Coron Artery Dis. 2000;11:77-81.

18. O'Donoghue M, de Lemos JA, Morrow DA, Murphy SA, Buros JL, Cannon CP, et al. Prognostic Utility of Heart- Type Fatty Acid Binding Protein in Patients With Acute Coronary Syndromes. Circulation. 2006;114:550-7.

19. Suzuki M, Hori S, Noma S, Kobayashi K. Prognostic Value of a Qualitative Test for Heart-Type Fatty Acid-Binding Protein in Patients With Acute Coronary Syndrome. Int Heart J. 2005;46:601-6.

20. McCann CJ, Glover BM, Menown IB, Moore MJ, McEneny J, Owens CG, et al. Prognostic value ofa multimarker approach for patients presenting to hospital with acute chest pain. Am J Cardiol. 2009;103:22-8.

21. Viswanathan K, Kilculten N, Morell C, Thistlethwaite SJ, Sivananthan MU, Hassan TB, et al. Heart-Type Fatty Acid-Binding Protein Predict LongTerm Mortality and Re-Infarction in Consecutive Patients With Suspected Acute Coronary Syndrome Who Are Troponin-Negative. J Am Coll Cardiol. 2010;55:2590-8.

22. Ishii J, Ozaki Y, Lu J, Kitagawa F, Kuno T, Nakano T, et al. Prognostic Value of Serum Concentration of Heart-Type Fatty Acid-Binding Protein Relative to Cardiac Troponin T on Admission in the Early Hours of Acute Coronary Syndrome. Clin Chem. 2005;51:1397-404. 